\title{
Educación en epidemiología para una medicina centrada en la persona.
}

\author{
Javier E. Saavedra ${ }^{1}$
}

\section{Resumen}

Tradicionalmente la enseñanza de la epidemiología se ha focalizado en desarrollar competencias en la investigación de la dinámica de las enfermedades con el objetivo de intentar comprenderlas, intervenirlas, limitarlas y especialmente prevenirlas. Sin embargo, existen propuestas que están enfatizando como fundamental para el cuidado de la salud, una atención al entendimiento de las circunstancias en torno a la misma y a la consideración de perspectivas de las personas acerca de sus problemas. Estas aproximaciones involucran la ampliación del ámbito del campo de la epidemiología a otros aspectos contextuales vinculados con conceptos de atención centrada en las personas o la gente, que involucran no solo aspectos de enfermedad o aspectos "negativos" de la salud sino también a aspectos más relacionados con bienestar que han sido denominados salud positiva. Contribuciones desde la salud mental pero perfectamente aplicadas a la salud en general, anticipan el desarrollo de una epidemiología orientada hacia la formulación de una ciencia del bienestar y al avance hacia una promoción de la salud a nivel más elevado tanto en el ambiente clínico como en el de la salud pública. Por tal motivo, se propone que la enseñanza de la epidemiología integre dos aspectos, por un lado, incluir en la búsqueda de la exploración de la dinámica y entendimiento de las enfermedades, los factores relacionados con salud positiva; y por otro lado, integrar al del planteamiento clásico de un período prepatogénico y patogénico, un enfoque a la salud positiva que considere de la misma manera un período pre-salutogénico y salutogénico, contemplando no solo los factores protectores de la salud desde un modelo de enfermedad, sino la identificación de factores promotores e inhibidores de la salud, convirtiendo la salud positiva en centro de atención y de estudio epidemiológico. La combinación de ambos, enfermedad y bienestar o salud negativa y salud positiva, implica la ampliación a un modelo más comprehensivo de la salud vinculado y dirigido a la totalidad de las personas; de esta manera la actividad epidemiológica y por ende la atención clínica devienen en una verdadera epidemiología de la salud.

\section{Introducción}

La epidemiología es una de las principales herramientas de la salud pública y los cambios en las aproximaciones, tanto de la epidemiología como de la salud pública, ha generado una repercusión mutua y por lo tanto un impacto en el objeto de estudio y en los contenidos de la enseñanza epidemiológica ${ }^{(1)}$ Diversos aspectos han propiciado estos cambios, el primero de ellos fue el progresivo control y descubrimiento de las causas de las enfermedades infecciosas y el consecuente aumento de la expectativa de vida; por lo tanto, el foco de atención de la salud pública y de la epidemiología fue migrando desde la preocupación sobre la mortalidad hacia una preocupación sobre la morbilidad, lo cual resaltó la importancia de las enfermedades crónicas y motivó la inclusión de la carga de morbilidad y los años perdidos de vida saludables como indicadores de los problemas de salud ${ }^{(2)}$. A su vez, los problemas en el control de las enfermedades crónicas pusieron de manifiesto la importancia de los factores conductuales, los estilos de vida, y los problemas sociales con enfoques más sistémicos, ampliando el campo de estudio de la epidemiología a estos factores y propiciando que la salud pública considere otros campos de preocupaciones ${ }^{(3)}$. A esto debemos agregar que la introducción de nuevas herramientas estadísticas y la biología molecular facilitaron el análisis de nuevos factores ${ }^{(4)}$. A la par de estos avances, ha cobrado mayor importancia la comunidad y otros actores en el campo de las decisiones políticas $^{(5)}$, y a su vez los logros en la democracia han propiciado que los estados brinden una mayor consideración a la lucha contra la inequidad ${ }^{(6)}$.

\section{¿El fin de la era del enfoque en la enfermedad?}

La centralización del foco de atención en las enfermedades crónicas por parte de la salud pública y la epidemiología, en contraste con las enfermedades agudas, a propiciado la ampliación del campo de acción a un nivel más complejo, ya que las manifestaciones clínicas interactúan con diversos factores contextuales, incluyendo la pobreza y otras inequidades ${ }^{(7)}$. Algunos autores incluso han llegado a sugerir que el foco de atención de la epidemiología debería incluir la pobreza como uno de los objetivos de erradicación más importante ${ }^{(8)}$. Asimismo, se ha comprendido que el enfoque de la medicina centrada en enfermedades, en una relación lineal entre enfermedad y agente causal, no se acomoda a la realidad clínica que incluye la comorbilidad y la interacción con un contexto psicosocial, y termina fragmentando la persona, más aún si a cada enfermedad le sigue una guía clínica y por lo tanto falla en considerar a la persona en su integralidad y en controlar apropiadamente los problemas de salud ${ }^{(9)}$. Por otro lado, los

${ }^{\prime}$ Médico Psiquiatra, Doctor en Medicina, Magister en Gerencia de Proyectos y Programas Sociales, Profesor Principal de la Universidad Peruana Cayetano Heredia, Director Ejecutivo de la Oficina Ejecutiva de Apoyo a la Investigación y Docencia Especializada del Instituto Nacional de Salud Mental "Honorio Delgado-Hideyo Noguchi”. 
avances en recursos de la medicina, la mayor expectativa de vida y la consecuente alta prevalencia de enfermedades crónicas ha ido modificando el concepto utópico de salud de un estado de completo bienestar físico, psicológico y mental hacia una definición de salud más dinámica, como enfocarse en la resiliencia y en la habilidad de adaptarse, tener autosuficiencia ante los retos físicos, emocionales y sociales y reestablecer el equilibrio y el logro de un estado bienestar a pesar de las circircunstancias $^{(10)}$. Este nuevo enfoque epidemiológico integra otros temas que fijan su atención no solo en la enfermedad sino en punto vista más integrador que ha venido a definirse como salud positiva ${ }^{(11)}$, convirtiéndola en objeto de estudio y por ende de necesidad de ser incluida en la enseñanza de la epidemiología.

Por lo tanto, se pone en contraposición dos perspectivas o modelos de cuidados médicos, que podrían ser interpretados tanto desde la salud individual como desde la salud pública. Por un lado, el modelo orientado a la enfermedad focalizado en el diagnóstico, prevención y tratamiento de enfermedades o patologías discretas, con mecanismos fisiopatológicos subyacentes y dirigido al tratamiento de la enfermedad causal con énfasis en la sobrevida; y por otro lado, el enfoque dirigido a la prioridades y preferencias de la persona o de las comunidades, que asume que las condiciones mórbidas o enfermedades emergen de una interacción compleja de factores genéticos, ambientales, psicológicos y culturales, cuyo foco de tratamiento se dirige a factores modificables que impiden el alcances de los objetivos de salud de la persona y donde el manejo de síntomas y discapacidades son el foco de atención y donde los resultados clínicos estén determinados por la personas o la gente y donde la sobrevida es una más de las metas propuestas y no necesariamente las más valiosa ${ }^{(9)}$. Ante esto han aparecido propuestas de modelos de atención que se centran en un sistema de salud como un todo para combatir la fragmentación bajo el principio de la atención centralizada en el paciente ${ }^{(12)}$, y también el enfoque de la medicina centrada en la persona que se ocupa no solo de la enfermedad sino de otros aspectos relacionados al bienestar de las personas y a los logros de otras dimensiones de su vida, como la espiritualidad, autonomía ${ }^{(13)}$. Este último enfoque tiene que ver con temas relacionados al respeto de la individualidad y a los valores de las personas, a la atención de los contextos sociales y a tomar en cuenta las perspectivas y la participación de las personas en el cuidado de su salud con una atención al entendimiento de las circunstancias en torno a la enfermedad ${ }^{(14,15)}$, y que son compartidos por distintos enfoques de la salud centrada en las personas ${ }^{(16)}$. Otros autores han insistido en el desarrollo de una epistemología moral en la medicina que integre hechos y valores del ámbito de la realidad social y psicológica de las personas en búsqueda de ayuda ${ }^{(17)}$. Consideramos que la epidemiología debe integrar en su enseñanza, tópicos vinculados a las propuestas de este modelo integrado y personalizado y al de salud positiva que se relaciona con el mismo.

\section{Un nuevo concepto de salud}

Siguiendo la necesidad de ampliar la noción de salud a un campo más allá de la enfermedad, un estudio reciente evaluó un nuevo concepto de salud como 'la habilidad de adaptarse y ser autogestionario' entre diversos actores, para evaluar indicadores que lo puedan hacer medible ${ }^{(18)}$. Participaron 1,938 personas entre médicos, enfermeras, fisioterapeutas, pacientes con enfermedades crónicas, hacedores de políticas, aseguradoras, profesionales de salud pública, ciudadanos e investigadores de distintas ramas. El nuevo concepto fue apreciado especialmente porque consideraba que una persona es más que su enfermedad y porque enfatizaba las fortalezas de la gente en vez de sus debilidades, y ante la pregunta sobre cuáles eran los indicadores de salud, se identificaron 6 dimensiones: función corporal, función mental, dimensión espiritual o existencial, calidad de vida, participación social y comunitaria, y funcionamiento diario. Proponen los autores que estos indicadores reflejen el concepto de salud positiva. De ser así estos tópicos también podrían ser integrados en campo de la epidemiología como objeto de estudio y análisis. En este estudio también es interesante resaltar que entre los diferentes grupos de actores del proceso de atención (proveedores de servicios, pacientes, ciudadanos, hacedores de políticas, aseguradores e investigadores) la opinión sobre la importancia de la dimensión sobre función corporal era similar en todos ellos. Sin embargo, existió gran variabilidad en la importancia otorgada al resto de dimensiones. Por ejemplo, los hacedores de políticas, investigadores, y aseguradores tenían los puntajes más bajos en todo el resto de las dimensiones, pero especialmente en la dimensión espiritual, en la participación social dimensión y función mental. Asimismo, resulta preocupante que, entre los prestadores directos de salud como médicos, enfermeras, fisioterapeutas y pacientes, los médicos percibieron la menor importancia al resto de dimensiones fuera de la función corporal. En cambio, los pacientes tienden a valorar más los conceptos de salud positiva en comparación con los médicos. Es interesante que las enfermeras, quienes habitualmente están más cerca de los pacientes, dan importancia similar a estos indicadores, a diferencia de los médicos. Las enfermeras han cumplido un papel fundamental en el cuidado del paciente y en la consideración de aspectos que van más allá de la enfermedad y que han sido resaltados en un enfoque por su propia naturaleza centrada en la persona ${ }^{(19)}$. Los hallazgos de este estudio deben ser considerados si se plantea un cambio en el enfoque de la epidemiología y la consideración de nuevos conceptos.

\section{Hacia una epidemiología de la salud}

Por lo expuesto, la epidemiología está considerando literalmente sus raíces etimológicas, es decir el estudio de lo que sucede en las poblaciones pero en el sentido más integral y más cerca de los nuevos conceptos de salud, que considera no solo los proceso mórbidos o negativos del salud, sino también un contexto complejo donde participan otros eventos relacionados con la salud, como factores contextuales psicosociales y culturales, además de aspectos positivos como factores vinculados al bienestar, calidad de vida, y la consideración de la perspectiva de las personas o de la gente ${ }^{(20)}$. Esta aproximación a la salud negativa y a la salud positiva deviene en una epidemiología de la salud en su sentido más amplio orientada hacia la formulación de una ciencia del 
bienestar $^{(21)}$. La integración de nuevas variables para la investigación epidemiológica favorecerá el descubrimiento de dinámicas dirigidas a una promoción de la salud del más alto nivel $^{(22)}$.

La inclusión de la salud positiva en la epidemiología abre un nuevo panorama para una concepción más enriquecedora en la relación entre salud y enfermedad. Inicialmente el estado de bienestar psicológico se evaluaba en función a la presencia o ausencia de distrés, y la ausencia de distrés implicaba bienestar emocional; luego se empezó a evaluar directamente sentimientos positivos o negativos de bienestar $^{(23)}$. Korkeila ${ }^{(24)}$ en una revisión de indicadores para medir aspectos de salud mental auspiciada por la Comisión Europea para un sistema de monitoreo comprehensivo de la salud identificó bajo el dominio de la salud mental positiva los siguientes aspectos: sentimientos de felicidad, satisfacción por la vida, bienestar subjetivo, sentido de control personal, resiliencia, capacidad de afrontamiento al estrés, resistencia, sentido de coherencia, optimismo, empoderamiento, y calidad de vida. En los últimos años se está prestando atención a la felicidad como la forma más apropiada de medir el progreso social y el foco de atención de las políticas públicas ${ }^{(25)}$, pues esta se asocia con indicadores como el producto bruto interno, el soporte social o la expectativa de vida, entre otros ${ }^{(26)}$. Luego se han incluido dentro de los conceptos pertinentes a la promoción de la salud una variedad más compleja de dimensiones. Por un lado, como salud mental positiva: amor, intimidad, capacidad de relaciones recíprocas, empatía, inteligencia emocional, moderación, autorregulación, sabiduría, curiosidad y creatividad, coraje, y sentido de justicia; y por otro lado, la salud física positiva como aptitud o condición física ("estar en forma"), la capacidad de resiliencia ante lesiones, enfermedad o incapacidad; o vitalidad ${ }^{(27)}$. En ambos casos la salud positiva puede estar presente, aunque exista cierta pérdida funcional física o mental. La mayoría de estos aspectos se relacionan entre sí y a otros aspectos de comportamiento y contextuales de la persona. Mezzich ha definido la salud positiva como el espacio o aspectos que rodean a la patología o enfermedad completando de esta manera el concepto de salud en su sentido más amplio ${ }^{(20)}$. De esta manera la salud positiva promueve nuevas variables y líneas de estudio para una eventual mejoría en el entendimiento, tratamiento, prevención y de las enfermedades y promoción de la salud como se mencionó antes.

\section{Hacia un enfoque salutogénico}

La investigación epidemiológica y por ende la enseñanza en epidemiología debe plantear un enfoque más amplio de su campo de estudio que integre la búsqueda de la salud positiva como centro de atención y no solo la salud negativa, asumiendo un enfoque salutogénico, concepto introducido por Antonovsky en 1979 que plantea que la salud es un concepto relativo en un continuum y que la pregunta correcta no es qué causa la enfermedad si no qué origina la salud; lo que plantea una nueva dirección de la salud pública ${ }^{(28)}$. Esta perspectiva ha sido adoptada con entusiasmo en algunos lugares $^{(29)}$, algunos autores han llamado la atención sobre la poca importancia que le han brindado los investigadores y la necesidad de dirigir a una focalización en aspectos positivos del bienestar independientemente de la enfermedad ${ }^{(30)}$. Como se muestra en el gráfico 1 se plantea que, así como en la enfermedad se refiere a un período prepatogénico y a un período patogénico, en el caso de la salud positiva se considere un período presalutogénico y un período salutogénico, y a la vez integrar estos aspectos al análisis de la enfermedad.

\section{La integración de métodos cualitativos y cuantitativos: aportes de la antropología médica y la epidemiología cultural}

Otro de los cambios en los conceptos de enfermedad y salud pública proviene de la llamada epidemiologia cultural, la cual integra métodos de la antropología y la epidemiología y estudia aspectos etnográficos a través de las representaciones locales de salud y enfermedad ${ }^{(35)}$. La antropología médica y de la psiquiatría cultural se sostiene en tres conceptos fundamentales: a) las nociones de emic, posición desde la comunidad, por ejemplo las ideas locales de la enfermedad; y de etic, desde el sistema de salud, por ejemplo, los sistemas clasificatorios médicos; 2) la distinción entre las concepciones de 'dolencia', para describir la experiencia personal de enfermedad, en contraposición con los de 'enfermedad' para describir la interpretación médica de un problema de salud; y 3) el desarrollo de modelos explicativos de la enfermedad, la cual integra diferentes perspectivas de todos los involucrados en el proceso clínico en el intento de comprender el desarrollo de la enfermedad $^{(36)}$. Asimismo, invita a integrar el uso de narrativas o formulaciones idiográficas no solo en la práctica clínica sino también en el enfoque epidemiológico ${ }^{(37)}$. Lo narrativo recoge la vivencia total de la persona o de las comunidades no en la forma fragmentada si tomáramos en cuenta solo la enfermedad sino en su contexto total y se convierte en un complemento contextual que hace más justicia a los elementos culturales de la enfermedad y la dolencia, y ha sido propuesta como Formulación Idiográfica por el grupo de trabajo de Guías Internacionales de Evaluación Diagnóstica (IGDA) de la Asociación Mundial de Psiquiatría ${ }^{(38)}$. Esta formulación idiográfica se dirige a obtener de las personas con problemas de salud mental sus perspectivas en cuanto a la contextualización o causas de sus problemas, identificación de los recursos y aspectos positivos personales de los pacientes, y sus expectativas de atención por el sistema de salud.

En Latinoamérica, la Guía Latinoamericana de Diagnóstico Psiquiátrico (GLADP) incluyó la formulación idiográfica como parte de su formulación diagnóstica ${ }^{(39)}$; y un reciente estudio epidemiológico de salud mental realizado en zonas rurales de Perú utilizó dicha formulación en una encuesta mixta para obtener tanto información cuantitativa, como cualitativa $^{(40)}$. Para este último fin utilizó un cuestionario narrativo derivado de la formulación idiográfica y fue administrado a 235 personas adultas con problemas auto percibidos de salud mental. Utilizando metodología de análisis cualitativo el estudio encontró que con frecuencia se adjudica la causalidad de los problemas de salud mental a conflictos familiares y a pérdidas de personas significativas; además se 


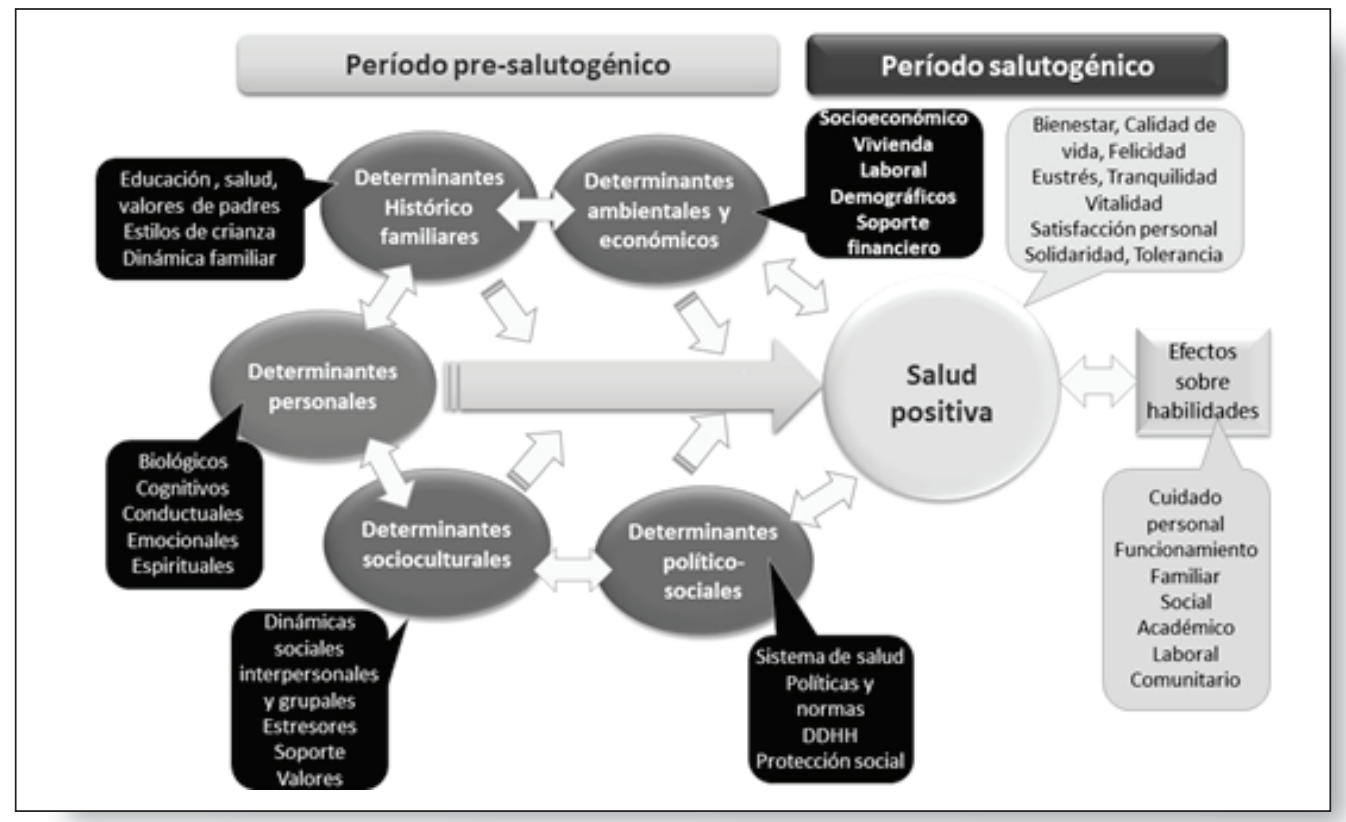

Elaborado y modificado a partir de Lahtinen E, Lethinen V, Riikonen E, Ahonen J, 1999. Framework for Promoting Mental Health in Europe ${ }^{(31)}$; Mezzich JE Üstün TB Quantitative and Experimental Methods in Psychiatry ${ }^{(32)}$; Korkeila Jyrki JA. Measuring aspects of mental health ${ }^{(33)}$; Palacios-Espinosa X \& Restrepo-Espinosa MH, $2008^{(3)}$.

Gráfico 1. Determinantes de la salud mental: el cómo, definiendo la estrategia de investigación y de intervención en salud positiva.

percibe escasos recursos comunitarios para enfrentar sus problemas de salud mental, y un grupo significativo desconoce qué esperar de los establecimientos de salud. Estos resultados ponen claramente en evidencia la alianza perversa entre problemas psicosociales y la poca presencia de la comunidad y sus recursos de salud para estas regiones. Por otro lado, también confirman la posibilidad de enriquecer el análisis epidemiológico cuantitativo con información de tipo cualitativo.

\section{La epidemiología centrada en la gente}

La equidad en ofrecer los mismos recursos a toda la población en realidad significa inequidad porque diferentes subgrupos de población tienen diferentes necesidades que podrían ser mejor captadas con enfoques centrados en la gente $^{(41)}$. La salud positiva lleva implícito la consideración de la persona y por extensión de la gente, haciendo justicia a la complejidad del ser humano y de las poblaciones, a la consideración del individuo y de la comunidad en su totalidad física, mental, social y cultural, y contribuye al avance de la ética en la atención de salud individual y de la salud pública ${ }^{(18,42)}$. La consideración de una epidemiología centrada en las personas favorece que se atienda a subgrupos de la población con necesidades y prioridades diferentes y por lo tanto el desarrollo de políticas sociales más justas. La enseñanza de la epidemiología debe tomar en cuenta que para resolver las inequidades que resultan en un espacio de gran diversidad como la nuestra debe haber una mirada a la cultura y a las particularidades de cada población y la atención a la salud positiva brinda esa posibilidad.

\section{Conclusiones}

Al lado de las enfermedades la enseñanza de la epidemiología debe brindar atención a los siguientes aspectos: a) preocupaciones de las personas sobre aspectos contextuales de las enfermedades (implicancias para su vida, creencias acerca de las enfermedades o los síntomas); b) prioridades de las personas acerca de su salud, valorizaciones de rendimiento personal con respecto a efectos colaterales; c) Determinantes causales no biológicos: estresores psicosociales, fuentes de soporte personal; d) Consideración de otros problemas interactuantes: salud mental, factores contextuales y culturales. La epidemiología debe utilizar enfoques amplios que incluyan una perspectiva integral de la salud y la enfermedad. Debe combinar aspectos positivos y negativos, buscando un enfoque para un diagnóstico de la salud de las poblaciones en su concepción más amplia. La epidemiología en salud debe buscar no solo factores de riesgo, protección y etiología de las enfermedades, sino también factores asociados y "generadores" de aspectos positivos de la salud, vinculados a bienestar y calidad de vida. Asimismo, debe tomar en cuenta la perspectiva de las personas y estar atento a los contextos tales como, valores, expectativas, dinámicas del sistema de salud, relación médico-paciente. Por último, debe poder utilizar aproximaciones desde lo general a lo particular, es decir desde las descripciones categóricas y dimensionales hasta las narrativas o idiográficas $\mathrm{y}$, por lo tanto, integrar análisis cuantitativos y cualitativos. 
1. Khoury MJ, Lam TK, Ioannidis JP, Hartge P, Spitz MR, Buring JE, Chanock SJ, Croyle RT, Goddard KA, Ginsburg GS, Herceg Z, Hiatt RA, Hoover RN, Hunter DJ, Kramer BS, Lauer MS, Meyerhardt JA, Olopade OI, Palmer JR, Sellers TA, Seminara D, Ransohoff DF, Rebbeck TR, Tourassi G, Winn DM, Zauber A, Schully SD. Transforming epidemiology for 21 st century medicine and public health. Cancer Epidemiol Biomarkers Prev. 2013 Apr;22(4):508-16. doi: 10.1158/1055-9965.EPI-13-0146. Epub 2013 Mar 5.

2.Murray CJ, Lopez AD. Measuring the global burden of disease. N Engl J Med. 2013 Aug 1;369(5):448-57. doi: 10.1056/NEJMra1201534.

3.Susser M, Susser E. Choosing a future for epidemiology: II. From black box to Chinese boxes and eco-epidemiology. Am J Public Health. 1996;86(5):674-677.

4.Susser M, Susser E. Choosing a future for epidemiology: I Eras and paradigms. Am J Public Health. 1996;86(5):668-673.

5.Eylesa J, Brimacombeb M, Chaulkc P, Stoddartd G, Prangere T, Moasee O. What determines health? To where should we shift resources? Attitudes towards the determinants of health among multiple stakeholder groups in Prince Edward Island, Canada. Soc Sci \& Med. 2001;53:1611-1619.

6. Rezaeian M. Challenges of Epidemiologists of Developing Countries in the 21st Century. Acta Med Iran 2016;54(1):4-8.

7. Starfield B. The hidden inequity in health care. Int J of Equity in Health. 2011;(10:15 doi:10.1186/1475-9276-10-15)

8. Krieger $\mathbf{N}$. Why epidemiologists cannot afford to ignore poverty. Epidemiology. 2007 Nov; 18(6):658-63.

9. Tinetti ME, Fried T. The end of the disease era. Am J Med. $2004 ; 116(3): 179-185$.

10. Huber M, Knottnerus JA, Green L, van der Horst H, Jadad AR, Kromhout D, Leonard B, Lorig K, Loureiro MI, van der Meer JW, Schnabel P, Smith R, van Weel C, Smid H. How should we define health? BMJ. 2011;343:d4163. doi: 10.1136/bmj.d4163.

11. Magyary D. Positive mental health: a turn of the century perspective. Issues in Mental Health Nursing. 2002;23:331349.

12. Stange KC, Nutting PA, Miller WL, Jaén CR, Crabtree BF, Flocke SA, Gill JM. Defining and Measuring the PatientCentered Medical Home. J Gen Intern Med. 2010;25(6):601612.

13. Mezzich J, Snaedal J, van Weel C, Heath I. Toward PersonCentered Medicine: From Disease to Patient to Person. Mount Sinai Journal of Medicine. 2010;77:304-306.

14. Stewart M. Towards a global definition of patient centred care. The patient should be the judge of patient centred care. BMJ 2001;322:444-445.

15. Little P, Everitt H, Williamson I, Warner G, Moore M, Gould C, Ferrier K, Payne S. Observational study of effect of patient centredness and positive approach on outcomes of general practice consultations. BMJ 2001;323:908-911.

16. Hughes JC, Bamford C, May C. Types of centredness in health care: themes and concepts. Med Health care and Philos. 2008;11:455-463.

17. Tauber AI. Medicine and the Call for a Moral Epistemology. Perspective in Biology and Medicine 2005;48(1):42-53.

18.Huber M, van Vliet M, Giezenberg M, Winkens B, Heerkens Y, Dagnelie PC, Knottnerus JA. Towards a 'patient-centred' operationalisation of the new dynamic concept of health: a mixed methods study. BMJ Open. 2016 Jan 12;6(1):e010091. doi: 10.1136/bmjopen-2015-010091.

19. Ghebrehiwet T. Nurses and Person-Centred Care. The International Journal of Person Centered Medicine. 2011;1 (1):20-22.

20. Mezzich JE. Positive Health: Conceptual Place, Dimensions and Implications. Psychopathology 2005;39:177-179.

21. Cloninger R. Implications of comorbidity for the classifications of mental disorders: The need for a psychobiology of coherent; in Maj M, Gaebel W, et al (eds): Psychiatric Diagnosis and Classification. Chichester, Wiley, 2002.

22. Herrman H, Saxena S Moodie R (eds): Promoting Mental Health: Concepts, Emerging Evidence, Practice. Geneva, WHO, 2005.

23. McDowell I. Psychological well-being, in: Measuring Health, a guide to rating scales and questionnaires. Third edition, Oxford, Oxford University Press: New York 2006, pp 206-272. Obtenido de http://citeseerx.ist.psu.edu/viewdoc/ download?doi=10.1.1.452.900\&rep=rep1\&type $=$ pdf

24. Korkeila JJA. Measuring aspects of mental health. National Research and Development Centre for Welfare and Health, Helsinki 2000. Obtenido de http://ec.europa.eu/health/ ph_projects/1998/promotion/fp_promotion_1998_frep_ 11_a_en.pdf.

25. Helliwell J, Layard R, Sachs J. World Happiness Report 2017. [online] New York: Sustainable Development Solutions network. 2017 Available at: http://worldhappiness. report/ed/2016/ [Accessed 18 oct.2018].https://s3. amazonaws.com/happiness-report/2017/HR17.pdf

26. Helliwell JF, Huang H, Wang S. Chapter 2 The Social Foundations of World Happiness. En Helliwell, J., Layard, R., \& Sachs, J. (2017). World Happiness Report 2017 (online), New York: Sustainable Development Solutions Network.

27. Herrman H, Saxena S Moodie R (eds): Promoting Mental Health: Concepts, Emerging Evidence, Practice. Geneva, WHO, 2005

28. Lehtinen V, Ozamiz A, Underwood L, Weiss M. Chapter 4 The Intrinsic Value of Mental Health. En Herrman H, Saxena S Moodie R (eds): Promoting Mental Health: Concepts, Emerging Evidence, Practice. Geneva, WHO, 2005:46-58.

29. Rivera de los Santos F, Ramos Valverde $P$, Moreno Rodríguez C, Hernán García M. Análisis del modelo salutogénico en España: aplicación en salud pública e implicaciones para el modelo de activos en salud. Rev Esp Salud Pública 2011;85:129-139.

30. Mittelmark MB, Bull T. The salutogenic model of health in health promotion research. Glob Health Promot. 2013 Jun;20(2):30-8. doi: 10.1177/1757975913486684.

31.Lahtinen E, Lethinen V, Riikonen E, Ahonen J. Edit. 1999. Framework for Promoting Mental Health in Europe. Heklsinki: National Research and Development Center for Welfare and Health Ministery of Social Affairs and Health Obtenido de http://ec.europa.eu/health/ph projects / 1996/promotion/fp_promotion_1996_frep_15_en.pdf

32. Mezzich JE, Üstün TB. Quantitative and experimental methods in psychiatry. Sadock BJ, Sadock VA, editors, 8 . Edition, Philadelphia: Lippincott Williams \& Wilkins, 2005:663-664 
33. Korkeila JJA. Measuring aspects of mental health. National Research and Development Centre for Welfare and Health, Helsinki 2000. Obtenido de http://ec.europa.eu/health/ ph_projects/1998/promotion/

34. Palacios-Espinosa $X$, Restrepo-Espinosa MH. Aspectos conceptuales e históricos del sentido de coherencia propuesto por Antonovsky: ¿una alternativa para abordar el tema de la salud mental?. Informes Psicologicos. 2008;10(11):275-300.

35. Weiss MG. Cultural Epidemiology: An Introduction and Overview. Anthropology \& Medicine. 2001;8(1):5-29. doi:10.1080/13648470120070980.

36. Weiss MG. The Promise of Cultural Epidemiology. Taiwanese Journal of Psychiatry (Taipei). 2017;31(1):8-24.

37.Phillips J. Idiographic Formulations, Symbols, Narratives, Context and Meaning. Psychopathology 2005;38:180-184. doi: 10.1159/000086087

38.IGDA Workgroup, WPA. IGDA 8: Idiographic (personalised) diagnostic formulation. British Journal of
Psychiatry. 2003;182(suppl.45):55-57.

39. Sección APAL de Diagnóstico y Clasificación: Guía Latinoamericana de Diagnóstico Psiquiátrico 1ra Edición. Guadalajara: APAL; 2004.

40. Saavedra JE, Uchofen-Herrera V. Percepciones sobre la atención de salud en personas con problemas autoidentificados de salud mental en zonas rurales del Perú. Revista Peruana de Medicina Experimental y Salud Pública. 2016; 33(34):785793. Disponible en: https://rpmesp.ins.gob. pe/index.php/rpmesp /article/view/2566/2593 Fecha de acceso: 20 oct. 2018 doi:http://dx.doi.org/10.17843/ rpmesp.2016.334.2566.

41. Starfield B. The hidden inequity in health care, Int J of Equity in Health 2011 (10:15 doi:10.1186/1475-9276-10-15)

42. Rutz W, Fernandez M, Trivedi J. Social Perspective on Psychiatry for the Person. The International Journal of Person Centered Medicine. 2011;1(1):140-142.

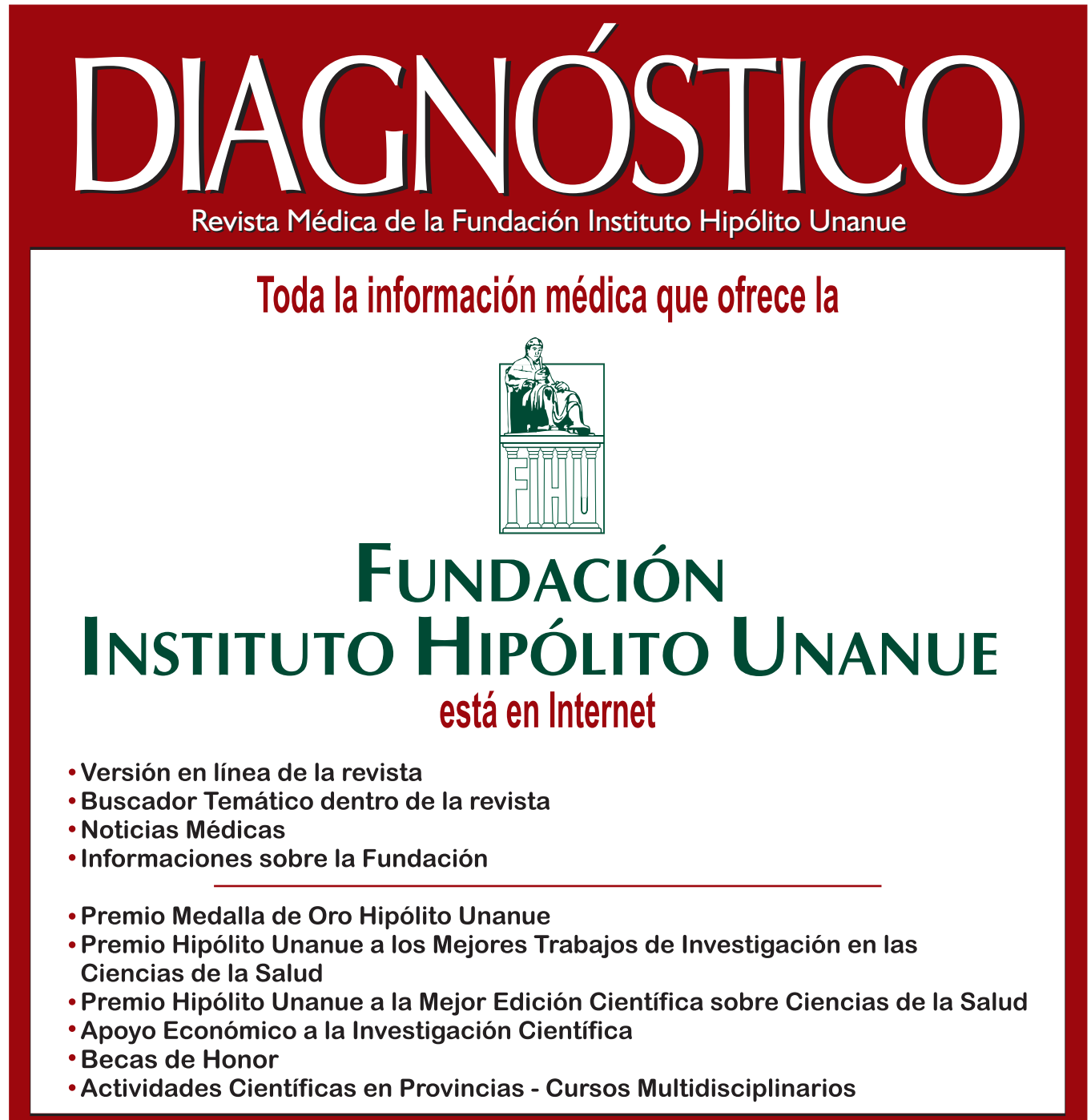

http://www.fihu-diagnostico.org.pe 